\title{
Symplectic reduction and topology for applications in classical molecular dynamics
}

\author{
F. J. Lin and J. E. Marsden \\ Department of Mathematics, University of California, Berkeley, California 94720
}

(Received 16 October 1991; accepted for publication 21 November 1991)

\begin{abstract}
This paper aims to introduce readers with backgrounds in classical molecular dynamics to some ideas in geometric mechanics that may be useful. This is done through some simple but specific examples: (i) the separation of the rotational and internal energies in an arbitrarily floppy $N$-body system and (ii) the reduction of the phase space accompanying the change from the laboratory coordinate system to the center of mass coordinate system relevant to molecular collision dynamics. For the case of two-body molecular systems constrained to a plane, symplectic reduction is employed to demonstrate explicitly the separation of translational, rotational, and internal energies and the corresponding reductions of the phase space describing the dynamics for Hamiltonian systems with symmetry. Further, by examining the topology of the energy-momentum map, a unified treatment is presented of the reduction results for the description of (i) the classical dynamics of rotating and vibrating diatomic molecules, which correspond to bound trajectories and (ii) the classical dynamics of atom-atom collisions, which correspond to scattering trajectories. This provides a framework for the treatment of the dynamics of larger $N$-body systems, including the dynamics of larger rotating and vibrating polyatomic molecular systems and the dynamics of molecule-molecule collisions.
\end{abstract}

\section{INTRODUCTION}

Basic interests in molecular physics include the study of (i) the structure and dynamics of molecules, clusters, and complexes (such as hydrogen-bonded complexes and van der Waals molecules) and (ii) the dynamics of molecular collisions, including the cases of atom-atom elastic scattering, atom-molecule inelastic scattering, and atom-molecule reactive scattering. While molecules naturally obey the laws of quantum mechanics, one fruitful approach has been to first examine the dynamics determined by the laws of classical mechanics. This has been true both for the case of rotating, vibrating molecules ${ }^{1}$ and for the case of molecular collisions, ${ }^{2-8}$ including ionmolecule reactions. ${ }^{9}$ In fact, quantum mechanics was developed by assuming a correspondence with classical mechanics, ${ }^{10}$ and this is one of the directions in which the geometric approach to quantum mechanics has continued to develop. ${ }^{11-13}$ This approach builds upon a background in classical mechanics. ${ }^{14,12,15}$

Beginning with principles and examples that are familiar to classical molecular dynamicists, we will introduce some ideas from geometric mechanics. Specifically, we begin with Hamilton's equations in curvilinear coordinates and will show that they can be expressed geometrically in coordinate-free form in phase space. Other ideas to be discussed geometrically, include the separation of energies (cf. Ref. 16) accompanying the appropriate choice of a coordinate system, the description of dynamics in a moving coordinate system (cf. Ref. 17) in terms of dynamics on a reduced phase space, ${ }^{18}$ and the decoupling of the dynamics corresponding to the internal and rotational motions. ${ }^{19}$

These ideas will be motivated by looking specifically at (i) the dynamics of atomic clusters ${ }^{20}$ which are also relevant to the dynamics of van der Waals molecules, ${ }^{21}$ and (ii) the dynamics underlying atom-atom differential cross sections. ${ }^{3}$

Finally, we explicitly treat the two-body problem in a central force field potential. After geometrically deriving the separation of energies and the reduced phase spaces, we eventually look at the topology of the reduced phase spaces for the case of a molecular interaction potential. The reduced phase spaces of different topological types correspond to different physical situations, including the rotating, vibrating diatomic molecule and atom-atom elastic scattering.

This work provides a framework for the geometric treatment of the dynamics of $N$-body systems previously treated by other approaches, such as atom-diatom van der Waals complexes and atom-linear molecule van der Waals complexes (see, e.g., Ref. 22), or the dynamics of a diatomic molecule in a time-dependent potential, such as an electromagnetic field (see, e.g., Ref. 23). Another possible future area of application is in geometric computational quantum mechanical molecular dynamics studies. ${ }^{24}$

While the rotating, vibrating molecule has recently been examined geometrically, ${ }^{25,26}$ the previous results did not discuss the optimal decoupling of the internal and 
rotational dynamics. ${ }^{19}$ Interest has been expressed in the geometric approach to molecular collision dynamics ${ }^{27}$ and in the topology of the scattering dynamics. ${ }^{3}$ The topology of the Kepler problem has been examined previously. ${ }^{28-30}$ However, the earlier studies have not applied reduction techniques to the molecular problem, and the topological relationship between bound and scattering trajectories has not been discussed.

\section{HAMILTON'S EQUATIONS IN CURVILINEAR COORDINATES FOR THE STUDY OF CLASSICAL MOLECULAR DYNAMICS}

The Hamiltonian $H$ in curvilinear coordinates for a system with $N$ degrees of freedom is

$$
H=\frac{1}{2} \sum_{i=1}^{N} \sum_{j=1}^{N} g^{i j} p_{p} p_{j}+V,
$$

written in terms of the fundamental metric tensor $g_{i j}$ (Ref. 17) with inverse $g^{i j}$, i.e.,

$$
g^{i j}=g_{i j}^{-1}, \quad i, j=1,2, \ldots, N,
$$

momenta $p_{k}(k=1,2, \ldots, N)$, and potential energy $V$. Then Hamilton's equations take the explicit form

$$
\begin{aligned}
\dot{q}_{k} & =\frac{\partial H}{\partial p_{k}}, \\
& =\frac{\partial}{\partial p_{k}}\left(\frac{1}{2} \sum_{i=1}^{N} \sum_{j=1}^{N} g^{i j} p_{p_{j}}+V\right), k=1,2, \ldots, N, \\
\dot{p}_{k} & =-\frac{\partial H}{\partial q_{k}}, \\
& =-\frac{\partial}{\partial q_{k}}\left(\frac{1}{2} \sum_{i=1}^{N} \sum_{j=1}^{N} g^{i j} p_{i} p_{j}+V\right), k=1,2, \ldots, N .
\end{aligned}
$$

In the examples, we will treat cases where $g^{i j}$ is diagonal, i.e.,

$$
g^{i j}=\delta_{i j} g^{i i}, \quad i, j=1,2, \ldots, N,
$$

and the potential energy $V$ is a function of the coordinates $q_{k}(k=1,2, \ldots, N)$ alone. In these cases, Hamilton's equations simplify to

$$
\begin{aligned}
& \dot{q}_{k}=g^{k k} p_{k}, \quad k=1,2, \ldots, N \\
& \dot{p}_{k}=-\frac{\partial V}{\partial q_{k}}-\frac{1}{2} \sum_{i=1}^{N} \frac{\partial}{\partial q_{k}}\left(g^{i i}\right) p_{i}^{2}, \quad k=1,2, \ldots, N .
\end{aligned}
$$

When a change of variables to an appropriate curvilinear coordinate system such that $\dot{p}_{k}=0$ for some $k$ can be carried out, then one can reduce the dimensionality of the phase space describing the Hamiltonian system. This can be seen in the following simple example.

Polar coordinates

$$
\begin{aligned}
& x=r \cos \theta, \\
& y=r \sin \theta,
\end{aligned}
$$

are a special case of curvilinear coordinates with fundamental metric tensor $g_{i j}$ satisfying

$$
g_{i j}=\left(\begin{array}{cc}
1 & 0 \\
0 & r^{2}
\end{array}\right)
$$

and with corresponding inverse

$$
g^{i j}=\left(\begin{array}{cc}
1 & 0 \\
0 & \frac{1}{r^{2}}
\end{array}\right) .
$$

Thus, expressed in polar coordinates, the Hamiltonian $H$ for a system with a potential $V$ describing a central force field takes the form

$$
H\left(r, \theta, p_{r} p_{\theta}\right)=\frac{1}{2 m} p_{r}^{2}+\frac{1}{2 m} \cdot \frac{1}{r^{2}} p_{\theta}^{2}+V(r) .
$$

Then Hamilton's equations in this coordinate system are

$$
\begin{aligned}
& \dot{r}=\frac{\partial H}{\partial p_{r}}=\frac{1}{m} p_{r} \\
& \dot{p}_{r}=-\frac{\partial H}{\partial r}=-\frac{\partial V}{\partial r}+\frac{p_{\theta}^{2}}{m r^{3}}, \\
& \dot{\theta}=\frac{\partial H}{\partial p_{\theta}}=\frac{1}{m r^{2}} p_{\theta} \\
& \dot{p}_{\theta}=-\frac{\partial H}{\partial \theta}=0 .
\end{aligned}
$$

Hence the angular momentum $p_{\theta}$ is a constant and we obtain on the reduced phase space $\left(r, p_{r}\right)$ the reduced Hamiltonian $H_{p_{\theta}}\left(r, p_{r}\right)$, which we now express in the form

$$
H_{p_{\theta}}\left(r, p_{r}\right)=(1 / 2 m) p_{r}^{2}+V_{p_{\theta}}(r),
$$

with effective potential $V_{p_{\theta}}(r)$

$$
V_{p_{\theta}}(r)=V(r)+\left(1 / 2 m r^{2}\right) p_{\theta}^{2}
$$


parametrically dependent on $p_{\theta}$. This is the effective potential familiar to molecular dynamicists (see, for example, Refs. $6,3,7$, or 8 ). It is comprised of the original potential for the potential for the central force plus a potential energy term describing a centrifugal barrier. Hamilton's equations on the reduced phase space are

$$
\begin{aligned}
& \dot{r}=\frac{\partial H_{p_{\theta}}}{\partial p_{r}}=\frac{1}{m} p r \\
& \dot{p}_{r}=-\frac{\partial H_{p_{\theta}}}{\partial r}=-\frac{\partial V_{p_{\theta}}}{\partial r} .
\end{aligned}
$$

The reduced Hamiltonian has been employed with the appropriate mass $m$ (i) in the form given above by molecular collision dynamicists to describe the classical dynamics of atom-atom collisions $\mathbf{s}^{3,6,7}$ and (ii) in the corresponding quantum mechanical form by molecular spectroscopists to treat the rotating, vibrating diatomic molecule. ${ }^{31-33}$ In these cases, examples of molecular interaction potentials that have been employed include the Lennard-Jones ${ }^{34,35}$ potential and the Morse ${ }^{36}$ potential, respectively. Both of these potentials have the essential qualitative features of (i) attaining a very large positive value as the internuclear separation $r$ approaches 0 , (ii) attaining a finite negative value (the well depth) at the equilibrium internuclear separation, and (iii) vanishing as the internuclear separation tends to $\infty$, allowing the description of molecular dissociation.

The Lennard-Jones potential $V_{L J}(r)$ takes the form

$$
V_{\mathrm{LV}}(r)=4 \epsilon\left[(\sigma / r)^{12}-(\sigma / r)^{6}\right]
$$

where $\epsilon$ is the well depth and the potential vanishes at $r=\sigma$. The corresponding effective potential then takes the form

$$
V_{\mathrm{L}, p_{\theta}}(r)=V_{\mathrm{LU}}(r)+p_{\theta}^{2} / 2 m r^{2}
$$

This can be expressed in dimensionless form (called "reduced" units in the chemical physical literature). Following Hirschfelder, Curtiss, and Bird, ${ }^{6}$ we define the following variables in dimensionless units:

$$
\begin{aligned}
& r^{*}=r / \sigma, \\
& V^{*}=V / \epsilon, \\
& p_{\theta}^{*}=p_{\theta} /\left(2 m \epsilon \sigma^{2}\right)^{1 / 2} \\
& V_{p_{\theta}}^{*}=V_{p_{\theta}} / \epsilon
\end{aligned}
$$

Then, in dimensionless units, the effective potential corresponding to the Lennard-Jones potential is given by

$$
V_{\mathrm{L}, p_{\theta}}^{*}\left(r^{*}\right)=4\left[\left(r^{*}\right)^{-12}-\left(r^{*}\right)^{-6}\right]+p_{\theta}^{* 2} / r^{* 2}
$$

Above the value $p_{\theta}^{*}=1.569$, the effective potential has no inflection point and hence is purely repulsive for all values of $r^{*}$ (Ref. 6, p. 554; Ref. 7, p. 54).

The Morse potential $V_{M}(r)$ takes the form

$$
V_{\mathrm{M}}(r)=D e^{-2 a\left(r-r_{0}\right)}-2 D e^{-a\left(r-r_{0}\right)},
$$

where $D$ is the equilibrium dissociation energy, $r_{0}$ is the equilibrium internuclear separation, and $a$ is a positive constant determining the shape of the potential well. The corresponding effective potential is

$$
V_{\mathrm{M}, p_{\theta}}(r)=D e^{-2 a\left(r-r_{0}\right)}-2 D e^{-a\left(r-r_{0}\right)}+p_{\theta}^{2} / 2 m r^{2} .
$$

We now define the following variables in dimensionless units:

$$
\begin{aligned}
& r^{*}=r / r_{0}, \\
& V^{*}=V / D, \\
& a^{*}=a r_{0}, \\
& p_{\theta}^{*}=p_{\theta} /\left(2 m D r_{0}^{2}\right)^{1 / 2}, \\
& V_{p_{\theta}}^{*}=V_{p_{\theta}} / D .
\end{aligned}
$$

Then the dimensionless effective potential corresponding to the Morse potential is

$$
V_{\mathrm{M}, p_{\theta}}^{*}\left(r^{*}\right)=e^{-2 a^{*}\left(r^{*}-1\right)}-2 e^{-a^{*}\left(r^{*}-1\right)}+p_{\theta}^{* 2} / r^{* 2} .
$$

Note that the dimensionless effective potential corresponding to the Morse potential contains an adjustable parameter $a^{*}$ that determines the width of the potential well and that did not appear in the dimensionless effective potential corresponding to the Lennard-Jones potential. We choose $a^{*}=5$ so that the potential wells of $V_{M, p_{\theta}}^{*}\left(\mathrm{r}^{*}\right)$ and $V_{L J, p_{\theta}}^{*}\left(\mathrm{r}^{*}\right)$ have approximately the same width when plotted as a function of $r^{*}$. Numerically, we find that the value $p_{\theta}^{*}=1.413$ is the critical value for the Morse potential with $a^{*}=5$, i.e., for values of $p_{\theta}^{*}$ $\geqslant 1.413$, the effective potential is purely repulsive for all values of $r^{*}$.

These ideas also extend to larger $N$-body problems, such as the dynamics of atomic clusters (discussed in the next section), weakly bound van der Waals molecules, ${ }^{21}$ atom-atom collisions (discussed in Secs. IV and VI), and polyatomic reactions (discussed briefly in Sec. VII; see Ref. 8). As motivation for the more general and more 
mathematical treatment contained in Secs. V and VI, the next two sections (III and IV) each present a physical example followed immediately by its corresponding geometric treatment.

\section{SEPARATION OF THE ENERGY OF ROTATION AND THE INTERNAL VIBRATIONAL ENERGY}

\section{A. Instantaneous rigid body momentum}

Jellinek and $\mathrm{Li}^{20}$ showed that the total kinetic energy of any nonrigid $N$-body system can be expressed as the sum of its "overall rotational energy," which we will call simply the rotational energy, and its internal vibrational energy. We recall their result in Eq. (3.8) below.

Let $\left\{\mathbf{r}_{i}: i=1, \ldots, N\right\}$ be the coordinates of the particles and $\left\{\mathbf{p}_{i}: i=1, \ldots, N\right\}$ be the momenta of the particles. A force-free rotation of a true rigid body is characterized by the time evolution of its angular velocity $\omega^{\text {rb }}(t)$. Let $I^{\mathrm{rb}}(t)$ be the instantaneous inertia tensor of the rigid body with respect to the lab-oriented system of coordinates (assumed for simplicity to be an inertial frame). The rigid body total angular momentum $L^{\text {rb }}$ in the lab frame is

$$
\mathbf{L}^{\mathrm{rb}}=\mathbf{I}^{\mathrm{rb}}(t) \cdot \omega^{\mathrm{rb}}(t)
$$

and is a constant of the motion. For a nonrigid system, one defines the instantaneous angular velocity $\omega_{L}^{\text {"rb" }}(t)$ as follows: The total angular momentum $L$ of the nonrigid system is the product of the instantaneous inertia tensor $\mathbf{I}(t)$ and the instantaneous angular velocity $\omega_{L}^{\text {"rb" }}(t)$ :

$$
\mathbf{L}=\mathbf{I}(t) \cdot \boldsymbol{\omega}_{L} \mathrm{rb} "(t) .
$$

Define the rigid body momentum $\mathbf{p}_{i}$ "rb"

$$
\mathbf{p}_{i}^{\text {"rb" }}=m_{i}\left(\omega_{L}^{\text {"rb" }} \times \mathbf{r}_{i}\right), \quad i=1, \ldots, N
$$

and the difference $\Delta \mathbf{p}_{i}$ between it and the momentum $\mathbf{p}_{i}$

$$
\Delta \mathbf{p}_{i}=\mathbf{p}_{i}-\mathbf{p}_{i}{ }^{\text {“tb" }}, \quad i=1, \ldots, N .
$$

Then the total vibrational angular momentum vanishes, i.e.,

$$
\sum_{i=1}^{N} \mathbf{r}_{i} \times \Delta \mathbf{p}_{i}=0
$$

because the total angular momentum of the system is given by

$$
\mathbf{L}=\sum_{i=1}^{N} \mathbf{r}_{i} \times \mathbf{p}_{i},=\sum_{i=1}^{N} \mathbf{r}_{i} \times \mathbf{p}_{i}^{\text {"rb" }}+\sum_{i=1}^{N} \mathbf{r}_{i} \times \Delta \mathbf{p}_{i}
$$

and the instantaneous rigid body angular momentum satisfies

$$
\sum_{i=1}^{N} \mathbf{r}_{i} \times \mathbf{p}_{i}^{\text {"rb" }}=L .
$$

Then the total kinetic energy of the nonrigid system is

$$
\sum_{i=1}^{N} \frac{\mathbf{p}_{i}^{2}}{2 m_{i}}=\sum_{i=1}^{N} \frac{\left(\Delta \mathbf{p}_{i}\right)^{2}}{2 m_{i}}+\sum_{i=1}^{N} \frac{\left(\mathbf{p}_{i}^{\text {"tb" }}\right)^{2}}{2 m_{i}}+\sum_{i=1}^{N} \frac{\mathbf{p}_{i}^{\text {"rb" }} \cdot \Delta \mathbf{p}_{i}}{m_{i}} \text {. }
$$

The last term is the Coriolis term (cf. Refs. 14 and 37)

$$
\begin{aligned}
\sum_{i=1}^{N} \frac{\mathbf{p}_{i} \text { "rb" } \cdot \Delta \mathbf{p}_{i}}{m_{i}} & =\sum_{i=1}^{N}\left(\omega_{L}^{\text {"rb" }} \times \mathbf{r}_{i}\right) \cdot \Delta \mathbf{p}_{i}, \\
& =\omega_{L}^{\text {"rb" }} \cdot \sum_{i=1}^{N} \mathbf{r}_{i} \times \Delta \mathbf{p}_{i} \\
& =0
\end{aligned}
$$

which vanishes since the total vibrational angular momentum vanishes. Thus the total kinetic energy separates into two terms. The vanishing of the Coriolis term is crucial for the separation of energies in the general $N$ body case.

The identity (3.8) is used as one ingredient in the reduced energy-momentum method discussed below. While equation (3.8) separates the energy, it does not separate the dynamics. The block diagonalization results of Simo, Lewis, and Marsden ${ }^{19}$ do "separate" the dynamics near a relative equilibrium and will be sketched below.

\section{B. Application of the reduced energy-momentum method}

Let $Q$ be the configuration manifold and $P$ be the associated canonical phase space, i.e., the cotangent bundle $P=T^{*} Q$. The phase space is endowed with the canonical symplectic two-form $\Omega$. Let $H: P \rightarrow \mathbf{R}$ be a Hamiltonian function of the form kinetic plus potential energy . We assume that the Hamiltonian system possesses symmetry induced by a Lie group $G$, which acts on $P$ by canonical transformations. Let $\mathscr{G}$ be the Lie algebra of $G$. Let $\mathscr{G}^{*}$ denote the dual of the Lie algebra $\mathscr{G}$, and let $\mathbf{J}: P \rightarrow \mathscr{G}^{*}$ be the corresponding conserved quantity. The Hamiltonian $H: P \rightarrow \mathbf{R}$ can be expressed as ${ }^{19}$

$$
H(z)=\frac{1}{2}\left|p-p_{J}(z)\right|_{g^{-1}}^{2}+\frac{1}{2} \mathbf{J}(z) \cdot \mathscr{T}^{-1}(q) \mathbf{J}(z)+V(q)
$$

Here $p_{\mathrm{J}}(z)$ is the momentum associated with the "instantaneous angular velocity" $\xi(z)$ of the rotating body, i.e., the Legendre transformation FL of the instantaneous velocity field

$$
p_{\mathbf{J}}(z) \equiv \mathrm{FL}\left[\xi(z)_{Q}(q)\right] \in T_{q}^{*} Q,
$$


where $\xi_{Q}$ is the infinitesimal generator

$$
\xi_{Q}(q) \equiv \frac{d}{d e}[\exp (e \xi) \cdot q]_{e=0} \text { for }(\xi, q) \in \mathscr{G} \times Q
$$

$\mathscr{F}(q)$ is the instantaneous moment of inertia (also called the locked inertia tensor), which is related to the angular velocity $\xi$ and the angular momentum $\mu$ by $\mu=\mathscr{I}(q) \xi$ when $\mathbf{J}(z)=\mu . V(q)$ is the potential energy. The amended potential $V_{\mu}: Q \rightarrow \mathbf{R}$ (Refs. 29 and 12) satisfies

$$
V_{\mu}(q) \equiv V(q)+\frac{1}{2} \mu \cdot \mathscr{F}^{-1}(q) \mu .
$$

The shifted momentum $\tilde{p}$ is defined by

$$
\tilde{p}=p-p_{\mathrm{J}}(z) .
$$

Hence, the Hamiltonian can also be written

$$
H(z)=\frac{1}{2}|\widetilde{p}|_{g^{-1}}^{2}+V_{\mathbf{J}(z)}(q) .
$$

We now define the reduced Hamiltonian $H_{\mu}: P_{\mu}$ $\rightarrow \mathbf{R}$ on the reduced phase space $P_{\mu}=\mathrm{J}^{-1}(\mu) / G_{\mu}$, where $G_{\mu}$ is the isotropy subgroup of $\mu$, by

$$
H_{\mu}\left(z_{\mu}\right) \equiv \frac{1}{2}\left|p_{\mu}\right|_{g^{-1}}^{2}+V_{\mu}\left(q_{\mu}\right)
$$

for $z_{\mu}=\left(q_{\mu}, p_{\mu}\right)=\pi_{\mu}(q, \widetilde{p}) \in P_{\mu}$ in terms of the canonical projection $\pi_{\mu}: \mathrm{J}^{-1}(\mu) \rightarrow P_{\mu}$. In the reduced Hamiltonian $H_{\mu}\left(z_{\mu}\right)$, the kinetic energy of the original Hamiltonian appears as the sum of two decoupled terms: the rotational energy $\frac{1}{2} \mu \cdot \mathscr{I}^{-1}\left(q_{\mu}\right) \mu$, i.e., the energy associated with the total angular momentum $\mu$, in the amended potential and the internal vibrational energy $\frac{1}{2}\left|p_{\mu}\right|_{g^{-1}}^{2}$.

The second variation of the reduced Hamiltonian $D^{2} H_{\mu_{e}}\left(\widetilde{z}_{e}\right)$ at a relative equilibrium $\widetilde{z}_{e}=\left(q_{e}, 0\right) \in J^{-1}(0)$ is block diagonal ${ }^{19}$ with respect to certain spaces $\mathscr{S}_{\text {ORIG }}$, $\mathscr{W}_{\text {OINT, and }} \mathscr{W}_{\text {OINT }}^{*}$. The spaces $\mathscr{S}_{\text {ORIG }}$ and $\mathscr{W}_{\text {OINT }}$ of pure configuration variations are defined by lifting the elements of certain spaces $\mathscr{V}_{\text {RIG }}$ and $\mathscr{V}_{\text {INT }}$ to the space $\mathscr{S}_{0}$ of admissable variations of the reduced Hamiltonian

$$
\begin{aligned}
& \mathscr{S}_{\text {ORIG }} \equiv\left\{(\Delta q, 0): \Delta q \in \mathscr{V}_{\text {RIG }}\right\} \\
& \mathscr{F}_{\text {OINT }} \equiv\left\{(\delta q, 0): \delta q \in \mathscr{V}_{\text {INT }}\right\},
\end{aligned}
$$

where $\mathscr{V}$ is the space of admissible configuration variations

$$
\mathscr{V} \equiv\left\{\delta q \in T_{q_{e}} Q:\left\langle\delta q, \zeta_{Q}\left(q_{e}\right)\right\rangle_{g}=0 \quad \forall \xi \in \mathscr{G}_{\mu_{e}}\right\},
$$

and $\mathscr{V}$ is shown ${ }^{19}$ to be decomposed into two subspaces, the subspace $\mathscr{V}_{\text {RIG }}$ of rotational modes and the subspace $\mathscr{V}_{\text {INT }}$ of internal vibrational modes

$$
\mathscr{V}=\mathscr{V}_{\mathrm{RIG}} \oplus \mathscr{V}_{\mathrm{INT}} .
$$

The space $\mathscr{T r}_{\text {OINT }}^{*}$ of pure momentum variations is defined by lifting the element of the annihilator to the tangent space $\mathscr{G} \cdot q_{e}$ to the orbit $G \cdot q_{e}\left[\right.$ written $\left.\left(\mathscr{G} \cdot q_{e}\right)^{A}\right]$ to $\mathscr{S}_{0}$ :

$$
\mathscr{W}_{0 \mathrm{INT}}^{*} \equiv\left\{(0, \tilde{\delta p}): \tilde{\delta p} \in\left(\mathscr{G} \cdot q_{e}\right)^{A}\right\} .
$$

It is shown ${ }^{19}$ that near a relative equilibrium, the linearized dynamics describing the rotational and internal motions are expressible in a normal form.

\section{THE REDUCED PHASE SPACE FOR BEAM SCATTERING DIFFERENTIAL CROSS SECTION MEASUREMENTS}

\section{A. The laboratory to center of mass transformation}

Besides the analysis of experimental molecular spectroscopy results (see, for example, Ref. 21), another experimental approach for the determination of interatomic potentials is the analysis of crossed beam elastic scattering measurements. ${ }^{38,39,8}$ In order to interpret the results of such an experiment, the transformation of the coordinates from the laboratory (again assumed to be inertial) to the center of mass coordinate system ${ }^{4,5,14,8}$ is essential. Note that in the analysis of elastic scattering experiments, one uses the velocity phase space instead of the momentum phase space; this will be discussed further below.

Levine and Bernstein ${ }^{8}$ have outlined the setup: In a typical experiment, one crosses a beam of particles of mass $m_{1}$ and velocity $\mathbf{v}_{1}$ with a beam of particles of mass $m_{2}$ and velocity $v_{2}$. The beams intersect in a small region and the intensity of scattered particles of mass $m_{1}$ is measured as a function of the angle of deflection from the original beam direction.

The position of the center of mass $r_{c m}$ is defined by

$$
\left(m_{1}+m_{2}\right) \mathbf{r}_{\mathrm{cm}}=m_{1} \mathbf{r}_{1}+m_{2} \mathbf{r}_{2} .
$$

Thus the velocity of the center of mass $\mathbf{v}_{\mathrm{cm}}$ satisfies

$$
\left(m_{1}+m_{2}\right) \mathbf{v}_{\mathrm{cm}}=m_{1} \mathbf{v}_{1}+m_{2} \mathbf{v}_{2} .
$$

By conservation of total linear momentum, $\mathbf{v}_{\mathrm{cm}}$ is the constant velocity of the center of mass in the laboratory system.

Let $\mathbf{s}_{1}$ and $\mathbf{s}_{2}$ be the position of the particle of mass $m_{1}$ and $m_{2}$, respectively, in the center of mass system, i.e.,

$$
\begin{aligned}
& \mathbf{r}_{1}=\mathbf{r}_{\mathrm{cm}}+\mathbf{s}_{1} \\
& \mathbf{r}_{2}=\mathbf{r}_{\mathrm{cm}}+\mathbf{s}_{2} .
\end{aligned}
$$

Let $\mathbf{w}_{1}$ and $\mathbf{w}_{2}$ be the velocity of the particle of mass $m_{1}$ and $m_{2}$, respectively, in the center of mass system 


$$
\begin{aligned}
& \mathbf{v}_{1}=\mathbf{v}_{\mathrm{cm}}+\mathbf{w}_{1}, \\
& \mathbf{v}_{2}=\mathbf{v}_{\mathrm{cm}}+\mathbf{w}_{2} .
\end{aligned}
$$

Let the relative velocity $\mathbf{v}$ be defined to be

$$
\mathbf{\nabla}=\mathbf{v}_{2}-\mathbf{v}_{1} \text {. }
$$

Define $\mathbf{r}_{1}^{\prime}, \mathbf{r}_{2}^{\prime}, \mathbf{r}_{\mathrm{cm}}^{\prime}, \mathbf{v}_{1}^{\prime}, \mathbf{v}_{2}^{\prime}, \mathbf{v}_{\mathrm{cm}}^{\prime}, \mathbf{s}_{1}^{\prime}, \mathbf{s}_{2}^{\prime}, \mathbf{w}_{1}^{\prime}, \mathbf{w}_{2}^{\prime}$, and $\mathbf{v}^{\prime}$ to be the corresponding vectors after the collision. By conservation of total linear momentum, we have $\mathbf{v}_{\mathrm{cm}}^{\prime}=\mathbf{v}_{\mathrm{cm}}$ and hence $\mathbf{r}_{\mathrm{cm}}^{\prime}=\mathbf{r}_{\mathrm{cm}}+\mathbf{v}_{\mathrm{cm}} t$. For an elastic collision, the magnitudes of the initial and final velocities are equal, i.e., $\left|\mathbf{v}_{i}^{\prime}\right|=\left|\mathbf{v}_{i}^{\prime}\right|$. By looking at the velocities $\mathbf{v}_{1}^{\prime}$ and $\mathbf{v}_{2}^{\prime}$ after the collision in the laboratory system, it is not obvious whether a final velocity is due to a large impact parameter deflection (forward scattering in the center of mass system) or due to a head-on collision (rebound collision in the center of mass systcm). However, this can be readily determined by looking at the Newton diagram ${ }^{4,5}$ for the collision, i.e., by looking at the velocities $\mathbf{w}_{1}^{\prime}$ and $\mathbf{w}_{2}^{\prime}$ in the center of mass system after the collision.

Furthermore, after making the transformation to the center of mass coordinate system, the differential cross section $I(\theta)$, i.e., the intensity of deflections as a function of the scattering angle $\theta$, can then be expressed classically in terms of the impact parameter $b$ and the deflection function $\chi$ for one pair of colliding particles as

$$
I(\theta)=\frac{b}{\sin \theta|d \chi / d b|},
$$

with

$$
\chi=\pi-2 b \int_{r_{0}}^{\infty}\left(1-\frac{V(r)}{E}-\frac{b^{2}}{r^{2}}\right)^{-1 / 2} \frac{d r}{r^{2}}
$$

in terms of the classical turning point $r_{0}$, the intermolecular potential $V(r)$, and the total energy $E$. Hence, given an experimentally determined differential cross section, one can gain information about the intermolecular potential. While molecular interactions are quantum mechanical and the classical mechanical treatment includes singularities, e.g., at the glory angle and at the rainbow angle, the classical differential cross section provides important information about the intermolecular potential (see, for example, Ref. 8 for further discussion).

In this example, it is the velocity phase space (versus the momentum phase space) that is important. One first employs the well-known fact that the two-body central force problem can be reduced to a one-body problem. ${ }^{14}$ In order to determine the relationship between (i) the angle defined by the difference between the final and initial directions of the relative position vector between the particles and (ii) the scattering angle of one of the particles in the laboratory coordinate system, one must perform the above transformation between the laboratory and center of mass systems. Specifically, one must look at the velocities (instead of the momenta) in the center of mass system. This is due to the fact that, in the center of mass coordinate system, the total linear momentum is zero, and the two particles move, of course, with equal and opposite momenta. In the next subsection, we will give the geometric relationship between the initial velocity phase space and the reduced velocity phase space.

Note that, while equations (4.8) and (4.9) determining the differential cross section are derived by employing conservation of energy and conservation of angular momentum, they are not Hamilton's equations on the reduced phase space [cf. Eqs. (2.19) and (2.20)]. However, the energy and angular momentum dependence of the topology of the dynamics on the internal phase space will be discussed further below (cf. Sec. VI).

\section{B. Definition of tangent bundle reduction via the symplectic reduction theorem and the Legendre transformation}

We first summarize the results of application of the symplectic reduction theorem. ${ }^{18}$ For the $N$-body problem, let the configuration manifold $Q$ be $\mathbf{R}^{3 N}$ and let the phase space $P$ be the cotangent bundle $T^{*} Q=T^{*} \mathbf{R}^{3 N}$ with the canonical symplectic structure. Let the Lie group $G=\mathbf{R}^{3}$ act by translation on $Q=\mathbf{R}^{3 N}$ for each component of the configuration manifold, inducing an action by cotangent lifts on $T^{*} Q$, i.e., for $x \in \mathbf{R}^{3}$

$$
\mathbf{x} \cdot\left(\mathbf{q}_{1}, \ldots, \mathbf{q}_{\mathbf{N}}, \mathbf{p}_{1}, \ldots, \mathbf{p}_{\mathbf{N}}\right)=\left(\mathbf{q}_{1}+\mathbf{x}, \ldots, \mathbf{q}_{\mathbf{N}}+\mathbf{x}, \mathbf{p}_{1}, \ldots, \mathbf{p}_{N}\right)
$$

The momentum map is

$$
\mathbf{J}\left(\mathbf{q}_{1}, \ldots, \mathbf{q}_{\mathbf{N}}, \mathbf{p}_{1}, \ldots, \mathbf{p}_{\mathrm{N}}\right)=\mathbf{p}_{\mathbf{1}}+\cdots+\mathbf{p}_{\mathrm{N}}=\mathbf{p}_{0},
$$

corresponding to the total linear momentum. The reduced phase space $J^{-1}\left(\mathbf{p}_{0}\right) / G_{\mathbf{p}_{0}}$, where $G_{\mathbf{p}_{0}}$ is the isotropy subgroup of $p_{0}$ in $G$, is symplectically diffeomorphic to $T^{*} \mathbf{R}^{3(N-1)}$ with the canonical symplectic structure. Coordinates in the reduced phase space are often chosen to be Jacobi coordinates.

Furthermore, the dynamics can then be described on the reduced phase space. The Hamiltonian flow on the original phase space induces a Hamiltonian flow on the reduced phase space. The reduced Hamiltonian $H_{\mathbf{p}_{0}}$ on the reduced phase space is

$$
H_{\mathrm{p}_{0}} \circ \pi_{\mathrm{p}_{0}}=H \circ i_{\mathrm{p}_{0}}
$$

where $H$ is the $G$-invariant Hamiltonian on the original phase space, $\pi_{p_{0}}: \mathbf{J}^{-1}\left(\mathbf{p}_{0}\right) \rightarrow \mathbf{P}_{p_{0}}$ is the canonical projection and $i_{p_{0}}: \mathbf{J}^{-1}\left(\mathbf{p}_{0}\right) \rightarrow P$ is the inclusion. 
In order to define a tangent bundle reduction consistent with the above cotangent bundle reduction, we employ the Legendre transformation FL : $T Q \rightarrow T^{*} Q$, which relates the Lagrangian formulation on $T Q$ and the Hamiltonian formulation on $T^{*} Q .^{12}$ The tangent bundle reduction is defined so that the Legendre transformation commutes with reduction, i.e.,

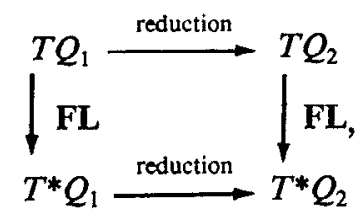

where $T^{*} Q_{2}=\left(T^{*} Q_{1}\right)_{P_{0}}$. In other words, one has

$$
T Q_{2} \equiv \mathbf{F L}-{ }^{-1}\left[\mathbf{F L}\left(T Q_{1}\right)\right]_{\mathbf{p}_{0}}
$$

Specifically, we have

$$
T \mathbf{R}^{3(N-1)} \equiv \mathbf{F L}-1\left(\left[\mathbf{F L}\left(T \mathbf{R}^{3 N}\right)\right]_{p_{0}}\right) .
$$

\section{SEPARATION OF ENERGIES AND REDUCED PHASE SPACES BY SYMPLECTIC REDUCTION: THE EXAMPLE OF TWO PARTICLES IN A CENTRAL FORCE FIELD}

We illustrate the separation of translational, rotational, and internal motions in the classical dynamics of two particles restricted to motion in a plane.

The configuration space $Q$ of the system is $\mathbf{R}^{2}$ $\times \mathbf{R}^{2}$. Coordinates on $Q$ are pairs $\left(\mathbf{r}_{1}, \mathbf{r}_{2}\right)$ of vectors in $\mathbf{R}^{2}$ describing the positions of the masses $m_{1}$ and $m_{2}$, respectively, in an inertial frame. Further, we will assume that $\mathbf{r}_{\mathbf{2}}-\mathbf{r}_{\mathbf{1}} \in \mathbf{R}^{2} \backslash\{\mathbf{0}\}$ so that the two particles do not occupy the same position in configuration space. The Lagrangian $L$ on the tangent bundle $T Q$ is given by

$$
L\left(\mathbf{r}_{1}, \mathbf{r}_{2}, \dot{\mathbf{r}}_{1}, \dot{\mathbf{r}}_{2}\right)=\frac{1}{2} m_{1}\left\|\dot{\mathbf{r}}_{1}\right\|^{2}+\frac{1}{2} m_{2}\left\|\dot{\mathbf{r}}_{2}\right\|^{2}-V\left(\left\|\mathbf{r}_{2}-\mathbf{r}_{1}\right\|\right),
$$

where we assume that the potential energy $V$ depends only on the distance $\left\|\mathbf{r}_{2}-\mathbf{r}_{1}\right\|$ between masses $m_{1}$ and $m_{2}$.

We apply the Legendre transformation to obtain the Hamiltonian $H$ on the cotangent bundle $T^{*} Q$

$H\left(\mathbf{r}_{1}, \mathbf{r}_{2}, \mathbf{p}_{1}, \mathbf{p}_{2}\right)=\frac{\left\|\mathbf{p}_{1}\right\|^{2}}{2 m_{1}}+\frac{\left\|\mathbf{p}_{2}\right\|^{2}}{2 m_{2}}+V\left(\left\|\mathbf{r}_{2}-\mathbf{r}_{1}\right\|\right)$.

The Euclidean group $\mathrm{E}(2)=\mathbf{R}^{2}$ (S) $\mathrm{SO}(2)$ acts on this system on $T^{*} Q$ by translation by and rotation about the center of mass of the system. In the case of identical particles, there is a further discrete symmetry $\mathbf{Z}_{2}$ corresponding to the interchange of the two particles.

\section{A. Separation of the translational energy from the sum of the rotational and internal energies}

The center of mass $r_{0} \in R^{2}$ of the system is defined by the equation

$$
\left(m_{1}+m_{2}\right) \mathbf{r}_{0}=m_{1} \mathbf{r}_{1}+m_{2} \mathbf{r}_{2} .
$$

The total linear momentum $\mathbf{p}_{0} \in \mathbf{R}^{2}$ of the system is a conserved quantity and satisfies the relationship

$$
\mathbf{p}_{0}=\mathbf{p}_{\mathbf{1}}+\mathbf{p}_{\mathbf{2}}
$$

Hence, we define the relative coordinate $\mathbf{r}$ and the relative momentum p:

$$
\begin{aligned}
& r \equiv \mathbf{r}_{2}-\mathbf{r}_{1} \\
& \mathbf{p} \equiv \mathbf{p}_{2}-\mathbf{p}_{1},
\end{aligned}
$$

and we obtain the following linear equations relating $\mathbf{r}_{\mathbf{1}}$, $\mathbf{r}_{2}, \mathbf{p}_{\mathbf{1}}$, and $\mathbf{p}_{\mathbf{2}}$ to $\mathbf{r}_{\mathbf{0}}, \mathbf{r}, \mathbf{p}_{\mathbf{0}}$, and $\mathbf{p}$ :

$$
\begin{aligned}
& \mathbf{r}_{1}=\mathbf{r}_{\mathbf{0}}+\left[m_{1} /\left(m_{1}+m_{2}\right)\right] \mathbf{r}, \\
& \mathbf{r}_{\mathbf{2}}=\mathbf{r}_{\mathbf{0}}-\left[m_{2} /\left(m_{1}+m_{2}\right)\right] \mathbf{r} \\
& \mathbf{p}_{\mathbf{1}}=\frac{1}{2} \mathbf{p}_{0}-\frac{1}{2} \mathbf{p} \\
& \mathbf{p}_{2}=\frac{1}{2} \mathbf{p}_{0}+\frac{1}{2} \mathbf{p} .
\end{aligned}
$$

We can transform from Cartesian coordinates $\left(\mathbf{r}_{1}, \mathbf{r}_{2}\right)$ to these coordinates $\left(\mathbf{r}, \mathbf{r}_{\mathbf{0}}\right)$ to recoordinatize the original phase space $\left(\mathbf{r}_{1}, \mathbf{r}_{2}, \mathbf{p}_{1}, \mathbf{p}_{2}\right)$ as the phase space $\left(\mathbf{r}, \mathbf{r}_{\mathbf{0}}, \mathbf{p}, \mathbf{p}_{0}\right)$ and then restrict to the reduced phase space $(\mathbf{r}, \mathbf{p})$ since $p_{0}$ is a constant and $r_{0}$ is a function of time satisfying

$$
\mathbf{r}_{0}(t)=\mathbf{r}_{0}(0)+\frac{1}{m_{1}+m_{2}} \mathbf{p}_{0} t
$$

This is an example of an equation describing a moving coordinate system or, equivalently, an equation describing a time-dependent holonomic constraint (cf. Ref. 17). This is the physical motivation for the first cotangent bundle reduction to follow now.

An element $\mathbf{a}$ of the translation group $\mathbf{R}_{2}$ acts on $Q$ by

$$
\Phi_{\mathbf{a}}\left(\mathbf{r}_{1}, \mathbf{r}_{2}\right)=\mathbf{a} \cdot\left(\mathbf{r}_{1}, \mathbf{r}_{2}\right)=\left(\mathbf{r}_{1}+\mathbf{a}, \mathbf{r}_{2}+\mathbf{a}\right),
$$

its tangent map $T \Phi_{\mathrm{a}}$ acts on $T Q$ by

$$
\begin{aligned}
T \Phi_{\mathrm{a}}\left(\mathbf{r}_{1}, \mathbf{r}_{2}, \dot{\mathbf{r}}_{1}, \dot{\mathbf{r}}_{2}\right) & =\left(\Phi_{\mathrm{a}}\left(\mathbf{r}_{1}, \mathbf{r}_{2}\right), D \Phi_{\mathbf{a}}\left(\mathbf{r}_{1}, \mathbf{r}_{2}\right) \cdot\left(\dot{\mathbf{r}}_{1}, \dot{\mathbf{r}}_{2}\right)\right) \\
& =\left(\mathbf{r}_{1}+\mathbf{a}, \mathbf{r}_{2}+\mathbf{a}, \dot{\mathbf{r}}_{1}, \dot{\mathbf{r}}_{2}\right),
\end{aligned}
$$

and hence the cotangent lift $T^{*} \Phi_{\mathrm{a}}$ defined by 


$$
\begin{aligned}
& T^{*} \Phi_{\mathrm{a}}\left(\mathbf{r}_{1}, \mathbf{r}_{2}, \mathbf{p}_{1}, \mathbf{p}_{2}\right) \cdot\left(\Phi_{\mathrm{a}}^{-1}\left(\mathbf{r}_{1}, \mathbf{r}_{2}\right),\left(\mathbf{p}_{1}, \mathbf{p}_{2}\right)\right) \\
& \quad=\left(\mathbf{r}_{1}, \mathbf{r}_{2}, \mathbf{p}_{1}, \mathbf{p}_{2}\right) \cdot T \Phi_{\mathrm{a}}\left(\Phi_{\mathrm{a}}^{-1}\left(\mathbf{r}_{1}, \mathbf{r}_{2}\right), \mathbf{p}_{1}, \mathbf{p}_{2}\right)
\end{aligned}
$$

acts on $T^{*} Q$ by

$$
T^{*} \Phi_{\mathbf{a}}\left(\mathbf{r}_{1}, \mathbf{r}_{2}, \mathbf{p}_{1}, \mathbf{p}_{2}\right)=\left(\mathbf{r}_{1}+\mathbf{a}, \mathbf{r}_{2}+\mathbf{a}, \mathbf{p}_{1}, \mathbf{p}_{2}\right)
$$

The corresponding momentum map $\mathbf{J}: T^{*} Q \rightarrow \mathbf{R}^{2}$ is defined by

$$
\mathbf{J}\left(\mathbf{r}_{1}, \mathbf{r}_{2}, \mathbf{p}_{1}, \mathbf{p}_{2}\right)=\mathbf{p}_{\mathbf{1}}+\mathbf{p}_{\mathbf{2}} \text {. }
$$

We start with the original phase space $T^{*} Q$ with the canonical symplectic structure $d \mathbf{r}_{1} \wedge d \mathbf{p}_{1}+d \mathbf{r}_{2} \wedge d \mathbf{p}_{2}$. The change of variables to the center of mass frame corresponds to a symplectic reduction, i.e., the quotienting of the inverse image (in the original phase space) of the constant total linear momentum $\mathbf{p}_{0}=\mathbf{p}_{1}+\mathbf{p}_{2}$ by translations $\mathbf{a}=\left[\mathbf{p}_{0} /\left(m_{1}+m_{2}\right)\right] \cdot t$. In other words, the reduced phase space at $\mathbf{p}_{0}$ is the quotient space

$$
\left(T^{*} Q\right)_{\mathbf{p}_{0}}=\mathbf{J}^{-1}\left(\mathbf{p}_{0}\right) / G_{\mathbf{p}_{0}}
$$

where $G_{\mathrm{p}_{0}}$ is the isotropy subgroup of $\mathrm{p}_{0}$ with respect to the coadjoint action of $R^{2}$ by cotangent lifts $T^{*} \Phi_{\mathrm{a}}$ on $T^{*} Q$. Further, by the cotangent bundle reduction theorem, one has the symplectic diffeomorphism

$$
\left(T^{*} Q\right)_{\mathrm{p}_{0}} \cong T^{*}\left(Q / G_{\mathrm{p}_{0}}\right) \text {. }
$$

Specifically, we have $T^{*}\left(\mathbf{R}^{2} \times \mathbf{R}^{2}\right)_{\mathbf{p}_{0}} \cong T^{*}\left(\mathbf{R}^{2} \backslash\{\mathbf{0}\}\right)$, where we have assumed $\mathbf{r}_{2}-\mathbf{r}_{1} \in \mathbf{R}^{2} \backslash\{0\}$. Further, the reduced phase space has the canonical symplectic structure $d \mathbf{r} \wedge d \mathbf{p}$. (The assumption of the atoms not occupying the same location in configuration space is implicitly understood in our notation for the original phase space.) The reduced Hamiltonian $H_{\mathrm{p}_{0}}$ on $T^{*}\left(\mathbf{R}^{2} \backslash\{\mathbf{0}\}\right)$ satisfies

$$
H_{\mathbf{p}_{0}}(\mathbf{r}, \mathbf{p})=\frac{\|\mathbf{p}\|^{2}}{2\left(m_{1} m_{2} /\left(m_{1}+m_{2}\right)\right)}+V_{\mathbf{p}_{0}}(\|\mathbf{r}\|)
$$

with amended potential $V_{\mathrm{p}_{0}}$ satisfying

$$
V_{\mathbf{p}_{0}}(\|\mathbf{r}\|)=V(\|\mathbf{r}\|)+\frac{\left\|\mathbf{p}_{0}\right\|^{2}}{2\left(m_{1}+m_{2}\right)} .
$$

In the new coordinatc system, Hamilton's equations become

$$
\begin{gathered}
\dot{\mathbf{r}}=\frac{\partial H_{\mathbf{p}_{0}}}{\partial \mathbf{p}}, \\
\dot{\mathbf{p}}=-\frac{\partial H_{\mathbf{p}_{0}}}{\partial \mathbf{r}},
\end{gathered}
$$

$$
\begin{aligned}
& \dot{\mathbf{r}}_{0}=\frac{\partial H_{\mathrm{p}_{0}}}{\partial \mathbf{p}_{0}}=\frac{1}{m_{1}+m_{2}} \mathbf{p}_{0}, \\
& \dot{\mathbf{p}}_{0}=-\frac{\partial H_{\mathbf{p}_{0}}}{\partial \mathbf{r}_{0}}=0,
\end{aligned}
$$

and $\mathbf{r}_{0}$ is a cyclic coordinate. Hence the dynamics can be described by the first two equations, the reduced Hamilton's equations. In other words, the dynamics drop to the reduced phase space $(\mathbf{r}, \mathbf{p})$.

\section{B. Separation of the rotational and internal energies}

For a central force potential, the angular momentum $p_{\theta}$

$$
p_{\theta}=x p_{y}-y p_{x}
$$

is a conserved quantity. We make the following change of variables from $(x, y) \in \mathbf{R}^{2} \backslash\{0\}$ to $(r, \theta) \in(0, \infty) \times S^{1}$ :

$$
x=r \cos \theta, \quad y=r \sin \theta,
$$

to transform from Cartesian coordinates $(x, y)$ to polar coordinates $(r, \theta)$ to recoordinatize the original phase space from its description in Cartesian coordinates $\left(x, y, p_{x}, p_{y}\right)$ to its description in polar coordinates $\left(r, \theta, p_{r} p_{\theta}\right)$. We then restrict to the reduced phase space $\left(r, p_{r}\right)$ since $p_{\theta}$ is a constant and the time dependence of $\theta$ is given by

$$
\theta(t)=\theta(0)+\omega t
$$

where $\omega$ is the angular velocity. Again, the expression for the eliminated coordinate [in this case, $\theta(t)$ ] is an equation describing a moving coordinate system or, equivalently, an equation for a time-dependent holonomic constraint.

As in the previous discussion for the initial reduction, an element $R_{\phi}$ of the group $\mathrm{SO}(2)$ acts on the configuration space $(0, \infty) \times S^{1}$ by

$$
\Phi_{R_{\phi}}(r, \theta)=R_{\phi} \cdot(r, \theta)=(r, \theta+\phi),
$$

its tangent map $T \Phi_{R_{\phi}}$ acts on $T\left((0, \infty) \times S^{1}\right)$ by

$$
\begin{aligned}
T \Phi_{R_{\phi}}(r, \theta, \dot{r}, \dot{\theta}) & =\left(\Phi_{R_{\phi}}(r, \theta), D \Phi_{R_{\phi}}(r, \theta) \cdot(\dot{r}, \dot{\theta})\right) \\
& =(r, \theta+\phi, \dot{r}, \dot{\phi}),
\end{aligned}
$$

and hence the cotangent lift $T^{*} \Phi_{R_{\phi}}$ defined by

$$
\begin{aligned}
T^{*} \Phi_{R_{\phi}}\left(r, \theta, p_{r} p_{\theta}\right) \cdot\left(\Phi_{R_{\phi}}^{-1}(r, \theta),(\dot{r}, \theta)\right) \\
=\left(r, \theta, p_{r} p_{\theta}\right) \cdot T \Phi_{R_{\phi}}\left(\Phi_{R_{\phi}}^{-1}(r, \theta), \dot{r}, \dot{\theta}\right)
\end{aligned}
$$


acts on $T^{*}\left((0, \infty) \times S^{1}\right)$ by

$$
T^{*} \Phi_{R_{\phi}}\left(r, \theta, p_{r} p_{\theta}\right)=\left(r, \theta+\phi, p_{r} p_{\theta}\right)
$$

The corresponding momentum map $\mathbf{J}_{\theta}: T^{*}((0, \infty)$ $\left.\times S^{\mathbf{l}}\right) \rightarrow \mathbf{R}$ is defined by

$$
\mathbf{J}_{\theta}\left(r, \theta, p_{r} p_{\theta}\right)=p_{\theta}
$$

The change of variables to the rotating frame corresponds to another reduction, specifically, the quotienting of the inverse image [in $\left.T^{*}\left((0, \infty) \times S^{1}\right)\right]$ of the constant total angular momentum $p_{\theta}=I \omega$, where $I$ is the moment of inertia, by rotations. The new reduced phase space is the quotient space

$$
T^{*}\left((0, \infty) \times S^{1}\right)_{p_{\theta}}=\mathbf{J}_{\theta}^{-1}\left(p_{\theta}\right) / G_{p_{\theta^{\prime}}}
$$

where $G_{p_{\theta}}$ is the isotropy subgroup of $p_{\theta}$ with respect to the coadjoint action of $\mathrm{SO}(2)\left(=S^{1}\right)$ by cotangent lifts $T^{*} \Phi_{R_{\phi}}$ on $T^{*}\left((0, \infty) \times S^{1}\right)$. In fact, again by the cotangent bundle reduction theorem, one has

$$
T^{*}\left((0, \infty) \times S^{1}\right)_{p_{\theta}} \cong T^{*}(0, \infty) .
$$

The reduced phase space has the canonical symplectic structure $d r \wedge d p_{r}$

The reduced Hamiltonian $H_{\mathrm{p}_{0}, p_{\theta}}$ on $T^{*}(0, \infty)$ satisfies

$$
H_{\mathfrak{p}_{0}, p_{\theta}}\left(r, p_{r}\right)=\frac{p_{r}^{2}}{2\left(m_{1} m_{2} /\left(m_{1}+m_{2}\right)\right)}+V_{\mathrm{p}_{0}, p_{\theta}}(r)
$$

where the amended potential $V_{\mathrm{p}_{0}, p_{\theta}}$ is given by

$$
\begin{aligned}
V_{\mathrm{p}_{0}, p_{\theta}}(r)= & V_{\mathrm{p}_{0}}(\|\mathbf{r}\|)+\frac{p_{\theta}^{2}}{2\left(m_{1} m_{2} /\left(m_{1}+m_{2}\right)\right) r^{2}} \\
= & V(\|\mathbf{r}\|)+\frac{\left\|\mathrm{p}_{0}\right\|^{2}}{2\left(m_{1}+m_{2}\right)} \\
& +\frac{p_{\theta}^{2}}{2\left(m_{1} m_{2} /\left(m_{1}+m_{2}\right)\right) r^{2}}
\end{aligned}
$$

After two reductions, the kinetic energy of the original Hamiltonian $H$ appears as the sum of three decoupled terms: the translational kinetic energy $\left\|\mathbf{p}_{0}\right\|^{2} / 2\left(m_{1}\right.$ $+m_{2}$ ) corresponding to the total linear momentum, the overall rotational kinetic energy

$$
\frac{p_{\theta}^{2}}{2\left(m_{1} m_{2} /\left(m_{1}+m_{2}\right)\right) r^{2}}
$$

corresponding to the total angular momentum, and the vibrational kinetic energy

$$
\frac{p_{r}^{2}}{2\left(m_{1} m_{2} /\left(m_{1}+m_{2}\right)\right)} .
$$

There is no Coriolis term in the reduced Hamiltonian.

Hamilton's equations in the newest reduced system become

$$
\begin{aligned}
& \dot{r}=\frac{\partial H_{\mathbf{p}_{0}, p_{\theta}}}{\partial p_{r}}, \\
& \dot{p}_{r}=-\frac{\partial H_{\mathbf{p}_{0}, p_{\theta}}}{\partial r}, \\
& \theta=\frac{\partial H_{\mathbf{p}_{0}, p_{\theta}}}{\partial p_{\theta}}=\frac{1}{\left(m_{1} m_{2} /\left(m_{1}+m_{2}\right)\right) r^{2}} p_{\theta}=\omega, \\
& \dot{p}_{\theta}=-\frac{\partial H_{\mathbf{p}_{0}, p_{\theta}}}{\partial \theta}=0
\end{aligned}
$$

and $\theta$ is a cyclic coordinate. Again, the dynamics can be described by the first two equations, and the dynamics has dropped to the reduced phase space $\left(r, p_{r}\right)$. This is an especially simple case of a general construction.

\section{Reduced phase spaces and Hamilton's equations}

The preceding two-step procedure exhibits two applications of the symplectic reduction theorem and the consequent theorem that the (reduced) dynamics is describable on the reduced phase space. Further, it is an example of a reduction by stages, in which the phase space is reduced by a semidirect product group. In this case, the semidirect product group is the Euclidean group, $E(2)=\mathbf{R}^{2}$ (S) SO(2). We now present the coordinate-free statements followed by their corresponding coordinatized forms.

Symplectic reduction theorem: ${ }^{18}$ Let $(P, \Omega)$ be a symplectic manifold on which there is a Hamiltonian left action of a Lie group $G$ with equivariant momentum map $\mathbf{J}: P \rightarrow \mathscr{G}^{*}$. Assume that $\mu \in \mathscr{G}^{*}$ is a regular value of $\mathbf{J}$ and that the isotropy subgroup $G_{\mu}$ under the coadjoint action acts freely and properly on $J^{-1}(\mu)$. Then the reduced phase space $P_{\mu}=\mathbf{J}^{-1}(\mu) / G_{\mu}$ is a manifold with symplectic form $\Omega_{\mu}$ satisfying

$$
\pi_{\mu}^{*} \Omega_{\mu}=i_{\mu}^{*} \Omega,
$$

where $\pi_{\mu}: \mathrm{J}^{-1}(\mu) \rightarrow P_{\mu}$ is the canonical projection and $i_{\mu}: \mathrm{J}^{-1}(\mu) \rightarrow P$ is inclusion.

Theorem on dynamics on reduced phase spaces: ${ }^{18}$ Let $H: T^{*} Q \rightarrow \mathbf{R}$ be a $G$-invariant Hamiltonian, i.e., $H \circ T^{*} \Phi_{g}=H$ for all $g \in G$. The flow $F_{t}$ of the Hamiltonian vector field $X_{H}$ leaves the set $\mathbf{J}^{-1}(\mu)$ invariant and commutes with the (induced) $G_{\mu}$-action on $\mathbf{J}^{-1}(\mu)$, so it induces a Hamiltonian flow $F_{t}^{\mu}$ on $\left(T^{*} Q\right)_{\mu}$ by $\pi_{\mu} \circ F_{t}$ 
$=F_{t}^{\mu_{0}} \pi_{\mu}$. The reduced Hamiltonian $H_{\mu}:\left(T^{*} Q\right)_{\mu} \rightarrow \mathbf{R}$ is defined (as above) by $H_{\mu} \circ \pi_{\mu}=H \circ i_{\mu}$. Further, the Hamiltonian vector fields $X_{H}$ and $X_{H_{\mu}}$ are related by the canonical projection $\pi_{\mu}$.

Next, we apply these coordinate-free ideas to derive the corresponding results in curvilinear coordinates: Let $(P, \Omega)$ be a symplectic manifold and $H: P \rightarrow \mathbf{R}$ be a given $C^{1}$ function called the Hamiltonian. Define the Hamiltonian vector field $X_{H}: P \rightarrow T P$ by the condition

$$
i_{X_{H}} \Omega=d H,
$$

where $i_{X_{H}} \Omega$ is the interior product of $X_{H}$ and $\Omega$, and $d H$ is the exterior derivative of $H$. Let $\left(q^{1}, \ldots, q^{N}, p_{1}, \ldots, p_{N}\right)$ be canonical coordinates for $\Omega$. Then

$$
\Omega=\sum_{i=1}^{N} d q^{i} \wedge d p_{i}
$$

and

$$
X_{H}=\left(\frac{\partial H}{\partial p_{i}},-\frac{\partial H}{\partial q^{i}}\right)
$$

Further, an integral curve of $X_{H}$ is a solution $(q(t), p(t))$ of Hamilton's equations

$$
\begin{aligned}
& \dot{q}^{i}=\frac{\partial H}{\partial p_{i}}, \quad i=1,2, \ldots, N, \\
& \dot{p}_{i}=-\frac{\partial H}{\partial q^{i}}, \quad i=1,2, \ldots, N .
\end{aligned}
$$

For the general $N$-body problem, the reduced Hamiltonian $H_{\mu}$ on the reduced phase space $\left(q_{\mu}, p_{\mu}\right) \in P_{\mu}$ $=\mathrm{J}^{-1}(\mu) / G_{\mu}$ can be determined by employing an explicit generalization of the momentum map $\mathbf{J}$ defined in the reduced energy-momentum method, as follows:

$$
H_{\mu}\left(z_{\mu}\right)=\frac{1}{2}\left|p_{\mu}\right|_{g^{-1}}^{2}+V_{\mu}\left(q_{\mu}\right),
$$

where $g$ is the relevant fundamental metric tensor, $p_{\mu}$ is the shifted momentum

$$
p_{\mu}=p-p_{\mathrm{J}}
$$

$V_{\mu}$ is the amended potential

$$
V_{\mu}\left(q_{\mu}\right)=V\left(q_{\mu}\right)+\frac{1}{2} \mu \mathscr{I}^{-1}\left(q_{\mu}\right) \mu,
$$

$\mathbf{J}$ is now the momentum map corresponding to the relevant total momentum of the $N$-body system, and $\mathscr{I}\left(q_{\mu}\right)$ is the relevant mass factor. For the first reduction, $\mu$ is the total linear momentum, $\mathbf{J}$ is the momentum map corresponding to the total linear momentum, $\mathscr{F}$ is the total mass, $p$ is the total momentum, and $p_{\mathrm{J}}$ is the total linear momentum (cf. Sections IV B and V A). For the second reduction, $\mu$ is the total angular momentum, $\mathbf{J}$ is the momentum map corresponding to the total angular momentum, $\mathscr{I}$ is the instantaneous moment of inertia, $p$ is the total momentum minus the total linear momentum, and $p_{\mathrm{J}}$ is the total angular momentum (cf. Sects. III B and $V B$ ).

Define the reduced Hamiltonian vector field $X_{H_{\mu}}$ : $P_{\mu} \rightarrow T P_{\mu}$ by

$$
i_{X_{H_{\mu}}} \Omega_{\mu}=d H_{\mu}
$$

where $\Omega_{\mu}$ and $H_{\mu}$ are determined by the symplectic reduction theorem. Further, $H_{\mu}$ can be explicitly determined as just described. Then an integral curve $\left(q_{\mu}(t), p_{\mu}(t)\right)$ of $X_{H_{\mu}}$ is a solution of the reduced Hamilton's equations

$$
\begin{aligned}
& \dot{q}_{\mu}{ }^{i}=\frac{\partial H_{\mu}}{\partial p_{\mu_{i}}}, \quad i=1,2, \ldots, N-1, \\
& \dot{p}_{\mu_{i}}=-\frac{\partial H_{\mu}}{\partial q_{\mu}{ }^{i}}, \quad i=1,2, \ldots, N-1 .
\end{aligned}
$$

As shown by the examples of the dynamics relevant to atomic clusters and the dynamics relevant to atomatom differential cross sections described in the previous sections, these techniques apply both to the dynamics of bound molecular systems and to the dynamics of molecular collisions. A framework for describing two-body molecular dynamics and including the dynamics of a (bound) diatomic molecule and the dynamics of atomatom scattering as special cases is described in the next section.

\section{GLOBAL TOPOLOGY OF THE ENERGY- MOMENTUM MAP FOR A MOLECULAR INTERACTION POTENTIAL}

We follow the approach of Smale, ${ }^{29}$ who examined the Kepler problem and made a global topological study of the energy-momentum map $\left(H \times J_{\theta}\right)_{T Q}: T Q \rightarrow \mathbf{R}^{2}$ defined on the tangent bundle $T Q$. We will consider the corresponding energy-momentum map $\left(H \times J_{\theta}\right)_{T^{*} Q}$ : $T^{*} Q \rightarrow \mathbf{R}^{2}$ defined on the cotangent bundle $T^{*} Q$. These maps are related by the Legendre transformation FL : $T Q \rightarrow T^{*} Q ;$ specifically,

$$
(H \times J)_{T Q}=(H \times J)_{T^{*} Q^{\circ}} \mathbf{F L} .
$$

For simplicity of notation, in the following we will omit the subscript $T^{*} Q$ on the energy-momentum map. We now consider the energy-momentum map defined on the cotangent bundle $T^{*} Q$ and start with the original phase space and make a global topological study of the energymomentum map $H \times J_{\theta} \times \mathbf{J}: T^{*}\left(M^{2} \times \mathbf{R}^{2}\right) \rightarrow \mathbf{R}$ 
$\times \mathbf{R} \times \mathbf{R}^{2}$ from the original phase space to the space where the energy map $H$ and the momentum maps $J_{\theta}$ and $\mathbf{J}$ take their values $E, p_{\theta}$, and $\mathbf{p}_{0}$, respectively. Here we have written $M^{2}$ for $\mathbf{R}^{2} \backslash\{0\}$. We consider the inverse images (or integral manifolds or "fibers") $I_{E, p_{\theta}, \mathrm{p}_{0}}=(H$ $\left.\times J_{\theta} \times \mathbf{J}\right)^{-1}\left(E, p_{\theta}, \mathbf{p}_{0}\right)$ with $\left(E, p_{\theta}, \mathbf{p}_{0}\right) \in \mathbf{R}^{4}$. Then the bifurcation set $\Sigma$ in $\mathbf{R}^{4}$ is defined to be the set of points in $\mathbf{R}^{4}$ over which the energy-momentum map fails (in the differentiable sense) to be locally trivial. The bifurcation set includes the critical values of the energy-momentum map, and on the bifurcation set the topological type of $I_{E, p_{\theta} \mathrm{p}_{0}}$ changes. The dynamical system on $T^{*}\left(M^{2} \times \mathbf{R}^{2}\right)$ induces a dynamical system on the reduced integral manifold $I_{E, p_{\theta}}=I_{E, p_{\theta} \mathrm{p}_{0}} / G_{\mathrm{p}_{0}}$. This is a consequence of the theorem on the dynamics on reduced phase spaces. ${ }^{18}$ The isotropy subgroup $G_{\mathbf{p}_{0}}$ of $\mathbf{p}_{0}$ is $\mathbf{R}^{2}$ for all values of $\mathbf{p}_{0} \in \mathbf{R}^{2}$. Hence, we have $I_{E, p_{\theta} \mathbf{p}_{0}}=I_{E, p_{\theta}} \times \mathbf{R}^{2}$ for all values of $\mathrm{p}_{0}$, and the topological type of $I_{E, p_{\theta} \mathbf{p}_{0}}$ is independent of $\mathrm{p}_{0}$.

The problem has now been simplified to the study of the reduced integral manifold $I_{E, p_{\theta}}$ and the (reduced) energy-momentum map $H \times J_{\theta}: T^{*} M^{2} \rightarrow \mathbf{R} \times \mathbf{R}$. We can write the bifurcation set $\Sigma$ as $\Sigma=\Sigma_{0} \subset \mathbf{R}^{2}$. We now choose the potential $V$ to be a molecular interaction potential. To be specific, we choose $V$ to be the Morse potential in dimensionless units with $a^{*}=5$. Then the bifurcation set $\Sigma_{0}$ in $\mathbf{R} \times \mathbf{R}$ is given by

$$
\Sigma_{0}=\Sigma_{1} \cup \Sigma_{2} \cup \Sigma_{3}
$$

with

$$
\begin{aligned}
& \Sigma_{1}=\left\{\left(E, p_{\theta}\right) \in \mathbf{R}^{2}: V_{p_{\theta}}\left(r_{\min }\right)=E \text { and } 0 \leqslant\left|p_{\theta}\right| \leqslant p_{\theta_{\max }}\right\} \\
& \Sigma_{2}=\left\{\left(E, p_{\theta}\right) \in \mathbf{R}^{2}: E=0\right\} \\
& \Sigma_{3}=\left\{\left(E, p_{\theta}\right) \in \mathbf{R}^{2}: V_{p_{\theta}}\left(r_{\max }\right)=E\right\}
\end{aligned}
$$

where $r_{\min }$ and (when it exists) $r_{\max }$ are functions of $p_{\theta}$ defined as follows:

$r_{\min }=\min _{r>0}\left\{r \in \mathbf{R}: V_{p_{\theta}}^{\prime}(r)=0, \quad V_{p_{\theta}}^{\prime \prime}(r)>0, \quad\right.$ and $\left.r<\infty\right\}$

$r_{\max }=\min _{r>r_{\min }}\left\{r \in \mathbf{R}: V_{p_{\theta}}^{\prime}(r)=0, \quad V_{p_{\theta}}^{\prime \prime}(r)<0, \quad\right.$ and $\left.r<\infty\right\}$,

and $p_{\theta_{\max }}$ is determined numerically to be 1.413 for the case $a^{*}=5$. The bifurcation set $\Sigma_{0}$ divides the $E, p_{\theta}$-plane into four regions (including one region with two components) with integral manifolds $I_{E, p_{\theta}}$ of different topological types. The bifurcation set is plotted in Fig. 1.

The real line $\mathbf{R}$ acts on $I_{E, p_{\theta}}$ as the dynamical group by restricting the dynamics on $T^{*} M^{2}$ because the energy $H$ is an integral. The group $\mathrm{SO}(2)$ acts on $M^{2}$ by rotations and induces an action by cotangent lifts on $T^{*} M^{2}$. The induced action of $\mathrm{SO}(2)$ on $T^{*} M^{2}$ leaves all of the functions $V, H$, and $J_{\theta}$ invariant as well as the integral manifolds $I_{E, p_{\theta^{*}}}$ (The angular momentum $J_{\theta}$ is also an integral.) The (induced) action of $\mathrm{SO}(2)$ commutes with the action of $\mathbf{R}$, so the action of the product group $S O(2)$ $\times \mathbf{R}$ is defined on $T^{*} M^{2}$ and on $I_{E, p_{\theta}}$ for each $\left(E, p_{\theta}\right) \in \mathbf{R}^{2}$.

Proposition: Let $\boldsymbol{M}^{2}=\mathbf{R}^{2}(\mathbf{0})$. Let $V$ be the Morse potential in dimensionless units with $a^{*}=5$. Let $\left(E, p_{\theta}\right) \in \mathbf{R}^{2}$ and $p_{\theta} \neq 0$. An integral manifold $I_{E, p_{\theta}}$ $\subset T^{*} M^{2}$ is the (not necessarily disjoint) union of homogeneous spaces of SO $(2) \times \mathbf{R}$, i.e., spaces of the form $(\mathrm{SO}(2) \times \mathbf{R}) / G_{i}$, where $i=1,2, \ldots, n$.

Proof: Given $\left(E, p_{\theta}\right) \in \mathbf{R}^{2}$ and $p_{\theta} \neq 0, \mathrm{SO}(2) \times \mathbf{R}$ acts transitively on each component of $I_{E, p_{\theta}} \subset T^{*} M^{2}$. We then apply an elementary theorem characterizing transitive actions (see, e.g., Ref. 40, p. 75): Let the group $G$ act transitively on a set $S$ and let $G_{i}$ be the isotropy subgroup of $x_{i}$ for $x_{i} \in S$. Then the action of $G$ on $S$ is equivalent to the action of $G$ on the coset space $G / G_{i}(i=1,2, \ldots, n)$. Letting $i$ label the components of $I_{E, p_{\theta}}$, we get $I_{E, p_{\theta}}=$ $\cup{ }_{i=1}^{n} G / G_{i}$.

Q.E.D.

Thus a component of an integral manifold is of one of the following topological types depending on the value of $\left(E, p_{\theta}\right) \in \mathbf{R}^{2}$. In order of increasing value of the energy $E$, the possible topological types of the integral manifolds $I_{E, p_{\theta}}$ in the different regions of the $\left(E, p_{\theta}\right)$-plane are (i) the empty set (when $n=0$ ):

$$
\begin{aligned}
& I_{E, p_{\theta}}=\phi \\
& \text { on } R_{1}=\left\{\left(E, p_{\theta}\right) \in \mathbf{R}^{2}: E<V_{p_{\theta}}\left(r_{\min }\right) \leqslant 0, \quad 0 \leqslant\left|p_{\theta}\right|<p_{\theta_{\max }}\right\} \\
& \qquad \cup\left\{\left(E, p_{\theta}\right) \in \mathbf{R}^{2}: E<0 \leqslant V_{p_{\theta}}\left(r_{\min }\right), p_{\theta_{\max }}<\left|p_{\theta}\right|\right\},
\end{aligned}
$$

(ii) a single point [when $n=1$ and $G_{1}=\mathrm{SO}(2) \times \mathbf{R}$ ]:

$$
I_{E, p_{\theta}}=\text { point if }\left(E, p_{\theta}\right)=\left(V_{p_{\theta}}\left(r_{\min }\right), p_{\theta}\right)=(-1,0) \text {, }
$$

(iii) a circle (when $n=1$ and $G_{1}=\mathbf{R}$ ):

$$
I_{E, p_{\theta}}=S^{1} \text { on } \Sigma_{1} \backslash\{(-1,0)\},
$$

(iv) a torus (when $n=1$ and $G_{1}=\mathbf{Z}$ ): 


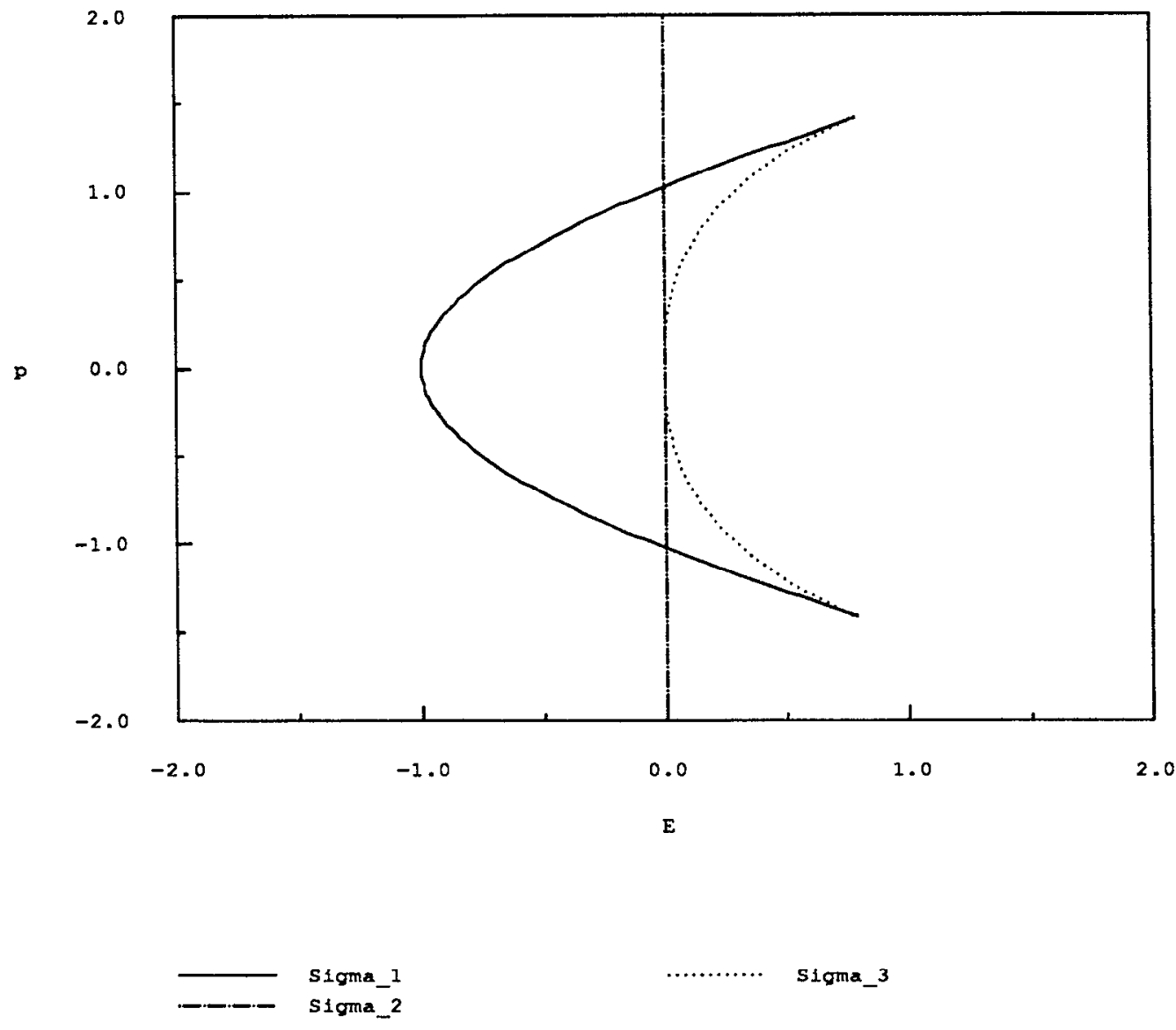

FIG. 1. The bifurcation set $\Sigma_{0}$ in the $E, p_{\theta}$-plane for the case of a Morse potential in dimensionless units with $a^{*}=5$.

$$
\begin{aligned}
& I_{E, p_{\theta}}=S^{1} \times S^{1} \\
& \quad \text { on } R_{2}=\left\{\left(E, p_{\theta}\right) \in \mathbf{R}^{2}: V_{p_{\theta}}\left(r_{\min }\right)<E<0,\right. \\
& \left.\quad 0 \leqslant\left|p_{\theta}\right|<p_{\theta_{\max }}\right\},
\end{aligned}
$$

(v) the union of a torus and a circle (when $n=2$, $G_{1}=\mathbf{Z}$, and $G_{2}=\mathbf{R}$ ):

$$
I_{E, p_{\theta}}=\left(S^{1} \times S^{1}\right) \cup S^{1} \text { on } \Sigma_{2},
$$

such that

$$
\left(S^{1} \times S^{1}\right) \cap S^{1}=\varnothing, \quad \text { if and only if } p_{\theta} \neq 0,
$$

(vi) the disjoint union of a torus and a cylinder (when $n=2, G_{1}=\mathbf{Z}$, and $G_{2}=I d$ ):

$$
\begin{aligned}
& I_{E, p_{\theta}}=\left(S^{1} \times S^{1}\right) \cup\left(S^{1} \times \mathbf{R}\right) \\
& \text { on } R_{3}=\left\{\left(E, p_{\theta}\right) \in \mathbf{R}^{2}: V_{p_{\theta}}\left(r_{\min }\right) \leqslant 0<E<V_{p_{\theta}}\left(r_{\max }\right),\right. \\
& \left.0<\left|p_{\theta}\right|<p_{\theta_{\max }}\right\},
\end{aligned}
$$

(vii) the union of a torus with a cylinder which intersect in a circle (when $n=2, G_{1}=\mathrm{Z}$, and $G_{2}=I d$ ):

$$
I_{E, p_{\theta}}=\left(S^{1} \times S^{1}\right) \cup\left(S^{1} \times \mathbf{R}\right) \text { on } \Sigma_{3} \text {, }
$$

with

$$
\left(S^{1} \times S^{1}\right) \cap\left(S^{1} \times \mathbf{R}\right)=S^{1},
$$

and (viii) a cylinder (when $n=1, G_{1}=I d$ ):

$$
I_{E, p_{\theta}}=S^{1} \times \mathbf{R}
$$

$$
\text { on } \begin{aligned}
R_{4} & =\left\{\left(E, p_{\theta}\right) \in \mathbf{R}^{2}: 0<V_{p_{\theta}}\left(r_{\max }\right)<E, \quad\left|p_{\theta}\right| \leqslant p_{\theta_{\max }}\right\} \\
& \cup\left\{\left(E, p_{\theta}\right) \in \mathbf{R}^{2}: 0 \leqslant E, \quad p_{\theta}=0\right\} \\
& \cup\left\{\left(E, p_{\theta}\right) \in \mathbf{R}^{2}: 0<E, \quad\left|p_{\theta}\right|>p_{\theta_{\max }}\right\} .
\end{aligned}
$$

A trajectory lying on a compact component of an integral manifold will be called a bound trajectory. A trajectory not contained in a compact subset of a noncompact component of an integral manifold will be called 
a scattering trajectory. This is consistent with the classification scheme of Goldstein. ${ }^{14}$

More specifically, scattering trajectories can be classified as grazing, (eventually) orbiting, or rebounding. A grazing trajectory lies in the noncompact component of an integral manifold of type (vi). An (eventually) orbiting trajectory initially lies on the cylinder and eventually lies on the circle in the integral manifold corresponding to $\Sigma_{3}$. A rebounding trajectory lies in the noncompact integral manifold of type (viii). This classification scheme is consistent with that familiar to molecular dynamicists (see, for example, Ref. 3, Fig. 12; Ref. 6, p. 556; or Ref. 7, p. 55; who each discussed the dynamics in terms of the Lennard-Jones potential). Further, our bifurcation set shown in Fig. 1 for the $\left(E, p_{\theta}\right)$-plane for a Morse potential is consistent with the description of the topology of scattering in the first quadrant of the $\left(p_{\theta}, E\right)$-plane for the Lennard-Jones potential as plotted by Ford and Wheeler ${ }^{3}$ Fig. 13) in terms of the dimensionless angular momentum and the dimensionless energy.

Finally, we restate the above results in terms of reduced phase spaces: A leaf $I_{E, p_{\theta}} / G_{p_{\theta}}$ of the reduced space $J_{p_{\theta}}^{-1}\left(p_{\theta}\right) / G_{p_{\theta}}$ is the (not necessarily disjoint) union of coset spaces of the form $\left((\mathrm{SO}(2) \times \mathbf{R}) / G_{x_{i}}\right) / S_{1}$, i.e., a point, a circle, or a line.

For a fixed value of $p_{\theta}$, the reduced space [( $\mathbf{J}$ $\left.\left.\times J_{\theta}\right)^{-1}\left(\mathrm{p}_{0}, p_{\theta}\right) / G_{\mathbf{p}_{0}}\right] / G_{p_{\theta}}$ is foliated by leaves, which are parameterized by $E$ and are the union of components of the following topological types: a point, a circle, or a line.

(i) If $E<V_{P_{\theta}}\left(r_{\min }\right)$, then the leaf of the reduced phase space is the empty set.

(ii) If $E=V_{p_{\theta}}\left(r_{\min }\right)$, then the leaf of the reduced phase space is topologically a point.

(iii) If $V_{p_{\theta}}\left(r_{\min }\right)<E<0$, then the leaf of the reduced phase space is topologically a circle.

(iv) If $E=0$ and $V_{p_{\theta}}\left(r_{\text {max }}\right)>0$, then the leaf of the reduced phase space is topologically the disjoint union of a circle and a point.

(v) If $0<E<V_{p_{\theta}}\left(r_{\max }\right)$, then the leaf of the reduced phase space is topologically the disjoint union of a circle and a line.

(vi) If $E=V_{p_{\theta}}\left(r_{\max }\right)$, then the leaf of the reduced phase space is topologically the union of a circle and a tangent line.

(vii) If $E>V_{p_{\theta}}\left(r_{\max }\right)$, then the leaf of the reduced phase space is topologically a line.

A leaf topologically equivalent to (a) a point corresponds to a rigid diatomic molecule in case (ii) and to two stationary distantly separated atoms in case (iv), (b) a circle corresponds to the phase space trajectory of a vibrating diatom in cases (iii) and (v), (c) a line corresponds to the phase space trajectory of an elastic atomatom collision: a grazing collision in case ( $v)$, an orbiting collision in case (vi), and a rebounding collision in case (vii).

\section{CONCLUDING REMARKS}

For molecular $N$-body systems with $N \geqslant 3$, the framework outlined above provides a starting point for the discussion of the topology of the dynamics, which may again be classified in terms of bound or scattering trajectories.

For the molecular three-body problem (we choose $N=3$ for simplicity), the scattering trajectories can be either reactive, i.e.,

$$
A+B C \rightarrow A B+C
$$

or

$$
A+B C \rightarrow A C+B,
$$

or nonreactive, i.e.,

$$
A+B C \rightarrow A+B C .
$$

Within each of these categories, the scattering trajectory can correspond to a collision that is either direct or complex (sometimes also called compound) ${ }^{8}$ In a direct reactive collision, the reaction takes place in a time shorter than one rotational period of the combined system, i.e., the time required for the reactants to rotate about each other. In a complex reactive collision, the reaction proceeds via a long-lived intermediate, i.e., a complex, which exists for more than one classical rotational period.

Hence, we make the following observations: Direct collisions generalize the scattering trajectories in the molecular two-body systems that are grazing trajectories or rebounding trajectories; complex collisions generalize the scattering trajectories in the two-body systems that contain orbiting trajectories; and complex collisions can also be considered to be a generalization of bound trajectories.

Application of the results regarding the decoupling of the reduced Hamiltonian at the linear level near a relative equilibrium ${ }^{19}$ imply that the dynamics of a molecular $N$ body system can be drastically simplified at the linear level near a relative equilibrium. The phase curves in the original phase space which project to equilibrium positions in the reduced system on the reduced phase space are called the relative equilibria of the original system. ${ }^{15}$ The results on simplifying the dynamics require the trajectory to be near a relative equilibrium, so we observe that physically the simplification of the molecular dynamics at the linear level requires the trajectory to be either a bound trajectory (for $N \geqslant 2$ ), an orbiting scattering trajectory (for $N=2$ ), or a complex scattering trajectory (for $N \geqslant 3$ ). (These conditions on the scattering trajectories are not necessarily sufficient.)

The geometric procedure outlined previously above provides a prescription for deriving reduced phase spaces 
that simplify the dynamics at the linear level near a relative equilibrium. It remains to extend this simplification of the dynamics to points other than relative equilibria.

The reduction of the phase space for an $N$-body system with $N \geqslant 3$ in $\mathbf{R}^{3}$ is more complicated than that discussed above for two-body systems in $\mathbf{R}^{2}$ when the total angular momentum $\mathbf{J}$ is nonzero.

This paper has presented two physical examples of molecular $N$-body systems for which symplectic reduction is relevant. In addition, for the case of $N=2$, we have presented a detailed, unified treatment of the planar dynamics. We showed that the two examples of the rotating, vibrating diatomic molecule and atom-atom elastic scattering are systems whose internal dynamics lie on reduced manifolds of different topological types. This description was unified by examination of the bifurcation set in the energy, angular momentum plane. Further, the above discussion outlines the relevance of this approach for the description of the dynamics of larger $N$-body systems.

${ }^{\text {I }}$ E. B. Wilson, Jr., J. C. Decius, and P. C. Cross, Molecular Vibrations (Dover, New York, 1980), republication of 1955 McGraw-Hill edition.

${ }^{2}$ K. W. Ford and J. A. Wheeler, Ann. Phys. (NY) 7, 259 (1959).

${ }^{3}$ K. W. Ford and J. A. Wheeler, Ann. Phys. (NY) 7, 287 (1959).

${ }^{4}$ D. R. Herschbach, The Vortex 22, 348 (1961).

${ }^{5}$ D. R. Herschbach, Discuss. Faraday Soc. 33, 149 (1962).

${ }^{6}$ J. O. Hirschfelder, C. F. Curtiss, and R. B. Bird, Molecular Theory of Gases and Liquids, second printing (Wiley, New York, 1964).

${ }^{7}$ R. E. Weston, Jr. and H. A. Schwarz, Chemical Kinetics (PrenticeHall, Englewood Cliffs, NJ, 1972).

${ }^{8}$ R. D. Levine and R. B. Bernstein, Molecular Reaction Dynamics and Chemical Reactivity (Oxford U. P., New York, 1987).

${ }^{9}$ B. H. Mahan, Accts. Chem. Res. 1, 217 (1968).

${ }^{10}$ B. Podolsky, Phys. Rev. 32, 812 (1928).

${ }^{11}$ A. Weinstein, Lectures on Symplectic Manifolds (American Mathematical Society, Providence, RI, 1977).

${ }^{12} \mathrm{R}$. Abraham and J. E. Marsden, Foundations of Mechanics (Benjamin/Cummings, Reading, MA, 1978), 2nd ed.
${ }^{13}$ V. Guillemin and S. Sternberg, Symplectic Techniques in Physics (Cambridge U. P., New York, 1984).

${ }^{14} \mathrm{H}$. Goldstein, Classical Mechanics (Addison-Wesley, Reading, MA, 1980), 2nd ed.

${ }^{15}$ V. I. Amold, Mathematical Methods of Classical Mechanics (Springer-Verlag, New York, 1978).

${ }^{16}$ C. Eckart, Phys. Rev. 47, 552 (1935).

${ }^{17} \mathrm{~L}$. Brillouin, Tensors in Mechanics and Elasticity (Academic, New York, 1964), first published in 1938 by Masson et Cie, Paris, France.

${ }^{18}$ J. E. Marsden and A. Weinstein, Rep. Math. Phys. 5, 121 (1974).

${ }^{19}$ J. C. Simo, D. Lewis, and J. E. Marsden, Arch. Rational Mech. Anal. 115, 15 (1991).

${ }^{20}$ J. Jellinek and D. H. Li, Phys. Rev. Lett. 62, 241 (1989).

${ }^{21}$ W. Klemperer, Faraday Discuss. Chem. Soc. 62, 179 (1977).

${ }^{22}$ K. R. Leopold, G. T. Fraser, F. J. Lin, D. D. Nelson, Jr., and W. Klemperer, J. Chem. Phys. 81, 4922 (1984).

${ }^{23}$ F. J. Lin and J. T. Muckerman, Comput. Phys. Commun. 63, 538 (1991).

${ }^{24}$ F. J. Lin, Bull. Am. Phys. Soc. 36, 1796 (1991).

${ }^{25}$ A. Guichardet, Ann. Inst. Henri Poincaré 40, 329 (1984).

${ }^{26}$ T. Iwai, Ann. Inst. Henri Poincaré 47, 199 (1987).

${ }^{27}$ A. Tachibana and T. Iwai, Phys. Rev. A 33, 2262 (1986).

${ }^{28}$ W. Kaplan, Am. Math. Month. 49, 316 (1942).

${ }^{29}$ S. Smale, Invent. Math. 10, 305 (1970).

${ }^{30} \mathrm{~S}$. Smale, Invent. Math. 11, 45 (1970).

${ }^{31}$ C. L. Pekeris, Phys. Rev, 45, 98 (1934).

${ }^{32}$ G. Herzberg, Molecular Spectra and Molecular Structure, Vol. I: Spectra of Diatomic Molecules (Van Nostrand Reinhold, New York, 1950), 2nd ed.

${ }^{33} \mathrm{C}$. H. Townes and A. L. Schawlow, Microwave Spectroscopy (Dover, New York, 1975), republication of 1955 McGraw-Hill edition.

${ }^{34}$ J. E. Jones, Proc. R. Soc. London Ser. A 106, 441 (1924).

${ }^{35}$ J. E. Jones, Proc. R. Soc. London Ser. A 106, 463 (1924).

${ }^{36}$ P. M. Morse, Phys. Rev. 34, 57 (1929).

${ }^{37}$ G. Herzberg, Molecular Spectra and Molecular Structure, Vol. II: Infrared and Raman Spectra of Polyatomic Molecules (Van Nostrand Reinhold, New York, 1945).

${ }^{38}$ J. M. Farrar, T. P. Schafer, and Y. T. Lee, in Transport Phenomena, edited by J. Kestin (A.I.P. Conference Proceedings, No. 11, 1973), p. 279.

${ }^{39}$ R. T Pack, J. J. Valentini, C. H. Becker, R. J. Buss, and Y. T. Lee, J. Chem. Phys. 77, 5475 (1982).

${ }^{40}$ N. Jacobson, Basic Algebra I, (Freeman, New York, 1985), 2nd ed.

${ }^{41}$ W. J. Satzer, Jr., Indiana Univ. Math. J. 26, 951 (1977).

${ }^{42} \mathrm{~J}$. E. Marsden, Lectures on Mechanics, London Mathematical Society Lecture Note Series (Cambridge U.P., New York, 1992) (to be published). 